\title{
3D Elemental Mapping with Nanometer Scale Depth Resolution via Electron Optical Sectioning
}

\author{
Timothy J. Pennycook ${ }^{\mathrm{a}, \mathrm{b}, 1, *}$, Hao Yang ${ }^{\mathrm{b}}$, Lewys Jones ${ }^{\mathrm{b}}$, Mariona Cabero $^{\mathrm{c}}$, Alberto Rivera-Calzada ${ }^{\mathrm{c}}$, Carlos Leon $^{\mathrm{c}}$, \\ Maria Varela ${ }^{c, d}$, Jacobo Santamaria ${ }^{c}$, Peter D. Nellist ${ }^{b, a}$ \\ ${ }^{a}$ EPSRC SuperSTEM Facility, Daresbury Laboratory, Warrington, WA4 4AD, UK \\ ${ }^{b}$ Department of Materials, University of Oxford, Parks Road, Oxford OX1 3PH, UK \\ ${ }^{c}$ Grupo de Fisica de Materiales Complejos, Universidad Complutense, 28040 Madrid, Spain \\ ${ }^{d}$ Materials Science $\&$ Technology Division, Oak Ridge National Laboratory, Oak Ridge, TN 37831, USA
}

\begin{abstract}
Electron energy loss spectroscopy in the scanning transmission electron microscope has long been used to perform elemental mapping but has not previously exhibited depth sensitivity. The key to depth resolution with optical sectioning is the transfer of sufficiently high lateral spatial frequencies. By performing spectrum imaging with atomic resolution we achieve nanometer scale depth resolution, enabling us to optically section an oxide heterostructure spectroscopically. Such 3D elemental mapping is sensitive to atomic scale changes in structure and composition and is more interpretable than Z-contrast imaging alone.
\end{abstract}

Keywords: STEM, EELS, Optical sectioning

\section{Introduction}

The advent of aberration correction in transmission electron microscopy (TEM) has brought about tremendous increases in image resolution, allowing the positions of individual atomic columns to be determined with up to picometer precision [1]. Such local information can prove vital to unlocking the physics behind new materials properties emerging from heterogenous structures such as interfaces, grain boundaries, impurities and other defect structures. A large proportion of the materials of interest today are three dimensional structures of heterogeneous elemental composition, hence a general method to directly determine three dimensional local atomic scale structural and elemental composition is highly desirable.

Bright-field phase-contrast imaging employed in conventional TEM instruments does not provide easily accessible compositional information. Scanning transmission electron microscopy (STEM) however provides chemically sensitive annular dark field (ADF) images. Rutherford scattering from the partially screened atomic nucleus causes the intensity of the ADF signal to increase with the atomic number $(Z)$ of the scattering element as approximately $\mathrm{Z}^{1.7}$, allowing the elemental composition of atomic columns and even individual atoms to be discerned directly from such Z-contrast images. Furthermore, aberration correction in STEM not only significantly increases

*Corresponding author. E-mail: tpennycook@gmail.com (T.J. Pennycook).

${ }^{1}$ Present address: Department of Physics, University of Vienna, Boltzmanngasse 5, 1090 Vienna, Austria. the lateral spatial resolution, but also provides a shallow depth of field. With just a few nanometers in optimal focus at a time, three dimensional information can be obtained by observing which features are in focus as the probe is focused to different depths. Such optical sectioning has previously been used with Z-contrast imaging in STEM to identify and determine the three dimensional positions of individual dopant atoms $[2,3,4,5,6,7]$, and to image the core structure and inclination of dislocations $[8,9,10]$. However, Z-contrast imaging suffers from an inability to differentiate heavier elements of similar atomic number and very light elements can be oiscured by the strong scattering of nearby heavy elements. Hence Z-contrast imaging alone is not sufficient for a general 3D compositional analysis method. For 2D compositional analysis where Z-contrast alone is insufficient, electron energy loss spectroscopy (EELS) is typically employed in conjunction with ADF imaging. Aberration correction has also enabled 2D elemental mapping to be performed with EELS at atomic resolution. Atomic resolution is a necessary condition for nanometer scale longitudinal depth resolution in STEM due to the hollow cone in the three dimensional optical transfer function (OTF) [11]. Operating in a confocal arrangement makes it possible to achieve depth dependent spectroscopic imaging at lower lateral resolution, but this requires significantly more complex and expensive instrumentation $[12,13]$. The ability to obtain atomic resolution compositional images with EELS now offers the tantalising prospect of spectroscopic optical sectioning without the need for confocal configurations. Furthermore, EELS and Z-contrast imaging can be performed 

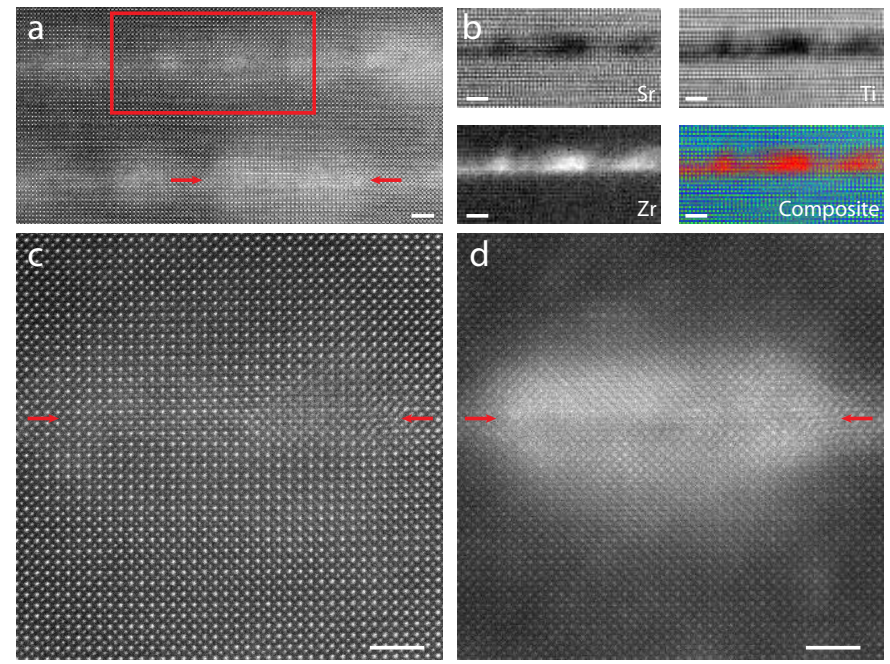

\section{d}
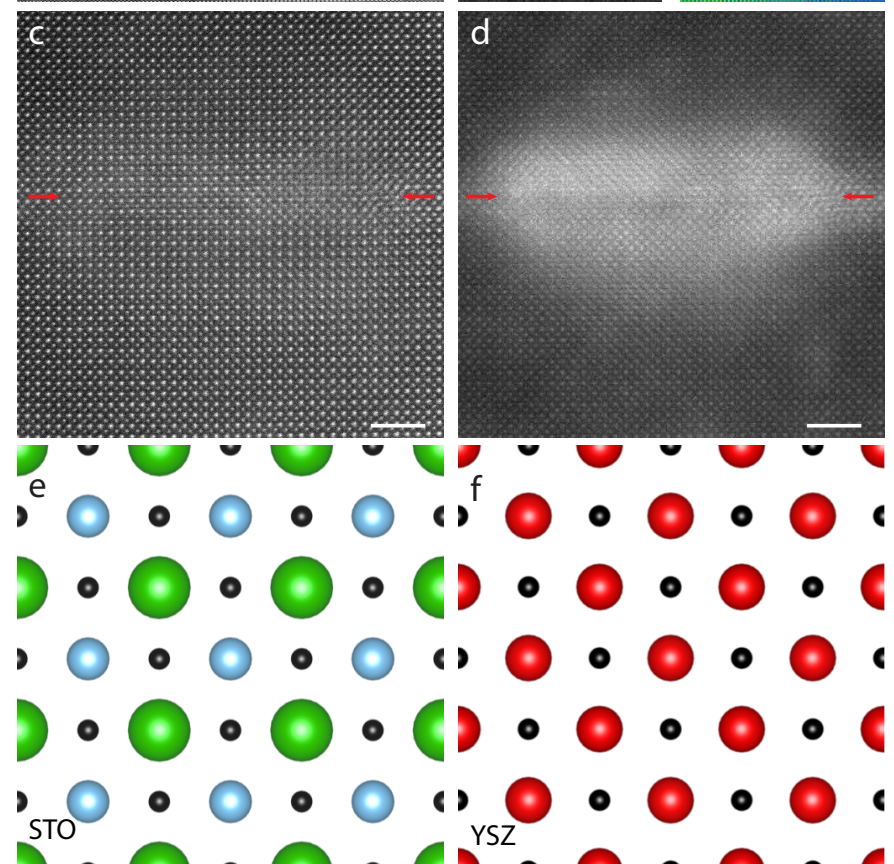

Figure 1: Wide field of view HAADF imaging of the YSZ/STO multilayers (a) shows bright layers which EELS elemental mapping (b) shows to be rich in $\mathrm{Zr}$ and $\mathrm{Sr}$ and $\mathrm{Ti}$ depleted. The maps shown in (b) were acquired from the region indicated by the red box in (a), and are displayed in the composite image with $\mathrm{Zr}$, Sr and Ti L-edges composing the red, green and blue channels respectively. (c) and (d) show high resolution HAADF and MAADF images of the region of the bright layer indicated by the red arrows in part (a). Models of the perovskite STO and cubic fluorite YSZ structures are shown in (e) and (f) respectively, viewed down the $\langle 100\rangle$ cubic axis with the cubic fluorite lattice matched to the STO. Zr, Sr, Ti and O are shown in red, green, blue and black respectively. Data acquired on a Nion UltraSTEM 200 at $200 \mathrm{kV}$. Scale bars indicate $2 \mathrm{~nm}$.

simultaneously and are complimentary, providing the ability to identify different sets of elements. Therefore, optical sectioning performed with both atomic resolution EELS and Z-contrast imaging methods offers a far more general 3D structural and compositional analysis tool than with Z-contrast alone. Theoretical simulations have shown that in principle optical sectioning can be performed with atomic resolution EELS spectrum imaging [14, 15, 16, but its experimental demonstration has not previously been achieved.

In this paper, we report the combined EELS and ADF optical sectioning of thin film heterostructures composed of yttria-stabilized zirconia $\left(\mathrm{Y}_{2} \mathrm{O}_{3}\right)_{x}\left(\mathrm{ZrO}_{2}\right)_{1-x}(\mathrm{YSZ})$ and $\mathrm{SrTiO}_{3}(\mathrm{STO})$, a system which has sparked intense debate due to the observation of colossal ionic conductivity at coherent YSZ/STO interfaces 17, 18, 19, 20, 21, 22, 23, 24, 25, 26, 27. Observing a sample with significant islanding, and hence a high level of overall inhomogeneity, we found regions which EELS shows to contain the elements of both YSZ and STO. ADF optical sectioning shows a change in contrast that suggests these regions are composed of YSZ buried beneath STO. Image simulations confirm that the observed contrast variation matches that expected for STO on top of YSZ, and also rule out the possibility that such a change of contrast could be caused just by channelling effects rather than a change in composition. Atomic resolution spectrum images acquired in these regions show a clear depth dependence that agrees with the changes seen in the ADF signal. In particular, a $\mathrm{Zr}$ lattice is resolved in spectroscopic optical slices acquired with the probe focused to depths exhibiting YSZ-like contrast in the ADF signal, but when the probe is focused to the entrance surface, where the ADF contrast appears STO-like, the $\mathrm{Zr}$ lattice blurs into an area of uniformly high intensity. The results establish the experimental feasibility of 3D elemental mapping with nanometer scale depth sensitivity, and highlight the advantages of spectroscopic optical sectioning. ADF through focal series can show depth dependent changes in composition, but elements of similar atomic number are often indistinguishable. In contrast 3D spectrum imaging provides unambiguous compositional analysis.

\section{Material and methods}

The STEM-EELS measurements were performed on a Nion UltraSTEM 100 operated at $100 \mathrm{kV}$ and equipped with an Enfina EEL spectrometer 28. Where noted, additional data were acquired on a Nion UltraSTEM 200 operated at $200 \mathrm{kV}$ and equipped with an Enfinium EEL spectrometer 29]. Both instruments utilise cold field emission electron sources, and correctors capable of neutralising up to fifth order aberrations. Image simulations were performed using the QSTEM package 30, employing 30 phonon configurations and parameters to match the experimental setup, i.e. a $100 \mathrm{kV}$ acceleration voltage, a 30 mrad convergence angle and a detector collection angle of 60-240 mrad

The YSZ/STO heterostructures were grown in a high oxygen pressure (3 mbar) pure O RF sputtering system, with the YSZ containing nominally $8 \mathrm{~mol}$ percent yittria. Samples were prepared for STEM observation in crosssection with conventional thinning and ion milling, and tilted in the microscope to the $\langle 100\rangle$ cubic zone axis of STO. The YSZ/STO system only grows in continuous thin films under very specific growth conditions. We have previously found that the deposition rate controls the crystalline orientation of the YSZ/STO system [23. High deposition rates favour mixed (110) and (100) growth giving rise to layers with typically disconnected polycrystalline islands, whilst low deposition rates promote (100) orientation and continuous layers. Even in samples with continuous layers, islands develop locally due to growth instabilities caused by the large $7 \%$ lattice mismatch. More often than not these islands are connected, and despite 
local islanding, continuous strained conduction pathways percolate between contacts allowing for the enhanced ionic transport. For this study we intentionally selected sample regions showing islands.

\section{Results and discussion}

Figure 1 illustrates the layered islanded nature of the sample with ADF and EELS data acquired on an UltraSTEM 200 alongside models of the STO and YSZ crystal structures. ADF imaging can distinguish STO from YSZ through the Z-contrast of their atomic columns [17, 22]. With atomic number 38, Sr appears considerably brighter than $\mathrm{Ti}$, atomic number 22 , as intensity in Z-contrast images scales as approximately the atomic number squared. Among such elements, the much lighter $\mathrm{O}$ atomic columns are not typically visible. STO therefore appears as an alternating pattern of bright and dark atomic columns corresponding to the Sr and Ti columns. When lattice matched to the STO lattice parameters, the cubic fluorite structure stabilised by the doping of yittria into zirconia appears the same as STO in projection, but with the $\mathrm{Sr}$ and $\mathrm{Ti}$ columns replaced with $\mathrm{Zr}$ columns as illustrated in Figure 1e-f. Since Sr, Y and Zr neighbour each other on the periodic table, ADF images of the YSZ will look very much like those of STO but with all the columns as bright as the $\mathrm{Sr}$ columns. Because of this the YSZ appears brighter overall than STO in Z-contrast images, and the regions appearing as brighter bands in the wide field of view high-angle ADF (HAADF) image shown in Figure 1 a can indeed be seen to contain Zr from EELS spectrum imaging (Figure 1p) using the integrated L-edges of $\mathrm{Sr}$, Ti and $\mathrm{Zr}$. The regions also exhibit strong strain contrast in medium angle ADF (MAADF) images (Figure 1 d ) as one would expect for YSZ layers strained to match the STO lattice. However the alternating pattern of bright and dark columns associated with STO can be seen to be continuous across these $\mathrm{Zr}$ containing regions in higher resolution HAADF images such as Figure 1k. The appearance of this pattern combined with the detection of significant $\mathrm{Ti}$ and $\mathrm{Sr}$ EELS signals in these layers may lead one to conclude that, rather than YSZ, the bright layers consist of a perovskite formed from an intermixture of Y, Zr, Sr and Ti.

However if one considers that the sample is not necessarily homogenous throughout its depth, an alternative possibility arises. Due to channelling, the clearest ADF images are formed when the probe is focused near the entrance surface, where the electron beam enters the sample. Combined with the fact that the depth of field in modern aberration corrected STEM instruments is only a few nanometers, this means one will tend to form images in which the entrance surface provides the dominant source of fine image details. The fine features of the material at depth will be blurred such that the lattice is not resolved. Therefore, if STO is at the entrance surface and YSZ is buried beneath, one would see STO like contrast in the individual atomic columns, but they will appear on top of
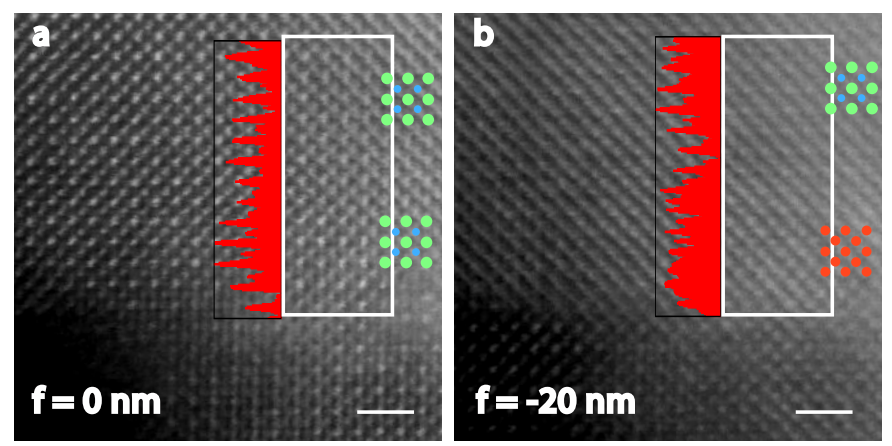

Figure 2: HAADF images from a through focal series of the islanded YSZ/STO multilayer system separated by a depth of approximately $20 \mathrm{~nm}$. The superimposed plots display the row-wise averaged intensities of the regions contained in the white boxes. Overlays are used to illustrate the local composition of the visible columns as indicated by the HAADF contrast at the top and bottom of these areas of interest, with $\mathrm{Sr}$ in green, $\mathrm{Ti}$ in blue and $\mathrm{Zr}$ in red. At the entrance surface (a) the alternating bright and dark rows of atomic columns of STO like contrast appears continuous from top to bottom in the image, with just a slight increase in the overall intensity appearing towards the bottom of the boxed region. $20 \mathrm{~nm}$ deeper into the film (b) the alternating rows of STO like contrast near the top of the boxed region transition into a region of similarly bright atoms in the bottom part of the boxed region showing no A-B site ordered contrast consistent with a buried coherent island of YSZ. Scale bars are $1 \mathrm{~nm}$.

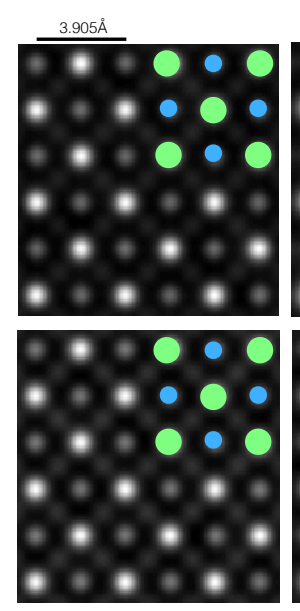

$\mathrm{f}=0 \mathrm{~nm}$
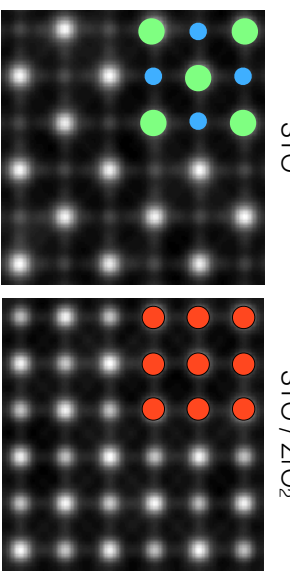

$f=-20 n m$

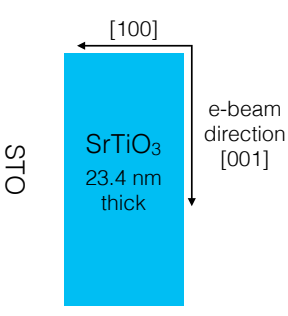

Figure 3: Comparison of HAADF images simulated with the probe focused to the entrance surface $(\mathrm{f}=0 \mathrm{~nm})$ and $20 \mathrm{~nm}$ further into the sample for two models, one composed entirely of STO (top row) and one of STO on top of $\mathrm{ZrO}_{2}$ (bottom row), as described in the text and illustrated schematically above. While the STO only slab shows considerable STO like contrast with the probe focused both to the entrance surface and $20 \mathrm{~nm}$ further into the depth of the sample, the $\mathrm{STO} / \mathrm{ZrO}_{2}$ model shows STO like contrast at the entrance surface and essentially YSZ like contrast at a depth of $20 \mathrm{~nm}$. Overlays are used to illustrate the structure of the visible heavy atoms in each optical slice, again with $\mathrm{Sr}$ in Green, $\mathrm{Ti}$ in blue and $\mathrm{Zr}$ in red. 
a background composed of an out of focus image of the YSZ. As every cation column in YSZ is similarly bright the out of focus image of the YSZ should appear as a diffuse region of higher intensity in the HAADF images, just as we observe in the regions EELS shows contain Zr.

In order to check for the presence of buried YSZ we performed simultaneous HAADF and MAADF optical sectioning in regions containing $\mathrm{Zr}$ rich layers. In the slices taken with the probe focused near the entrance of the sample, STO-like contrast appears continuous, except for a diffuse background of enhanced intensity in the $\mathrm{Zr}$ rich area as can be seen in Figure 2 a. This is particularly clear from the row averaged intensity plot, displayed as an inset, showing the overall intensity of the STO-like contrast increase towards the bottom of the region of interest. When the probe is defocused further into the sample the STO-like contrast disappears from this Zr rich region, becoming instead YSZ-like with every column having similar intensity. Interestingly, at the same depth, STO contrast persists outside the $\mathrm{Zr}$ rich area. An example of this can be seen in Figure 2b, which shows the same area as in Figure $2 \mathrm{a}$ but with the defocus set to approximately $20 \mathrm{~nm}$ deeper into the sample. A complete through focal series containing the regions shown in Figure 2 is available online.

Image simulations including the effects of channeling were performed to examine the possibility that channeling could be responsible for the change in contrast seen in the HAADF optical sectioning. A simple slab model was constructed consisting of a layer of STO on top of a layer of $\mathrm{ZrO}_{2}$ in the cubic fluorite structure lattice matched to the STO. Based on the HAADF optical sectioning, the total thickness of the slab was taken to be approximately 23 $\mathrm{nm}$, with STO taking up the top two thirds of the slab and $\mathrm{ZrO}_{2}$ the bottom third. A second slab model of the same thickness but composed entirely of STO was also created. Images simulated for both models exhibited strong STOlike contrast with the probe focused to the STO entrance surface. However the images simulated for the slab composed entirely of STO showed clear STO like contrast for all defoci, whereas the $\mathrm{STO} / \mathrm{ZrO}_{2}$ slab showed a change to zirconia like contrast when the probe was focused to the $\mathrm{ZrO}_{2}$ as illustrated in Figure 3. A small residual contrast remains on top of the $\mathrm{ZrO}_{2}$ contrast, but this would likely be difficult to observe with the presence of noise in an experiment. This corresponds to the experimental observation that the Zr-rich regions show a change from STO-like contrast to YSZ like contrast whereas regions not appearing to contain $\mathrm{Zr}$ show STO-like contrast at all depths.

A weakness of the ADF technique is an inability to distinguish between heavy elements of similar atomic number. From ADF imaging alone, the appearance of an area with every heavy column appearing similarly bright could be ascribed to the presence of a perovskite such as $\mathrm{SrZrO}_{3}$ (SZO). This can be ruled out in the present case from the optical sectioning data. The presence of continuous STO like contrast at the entrance surface is inconsistent
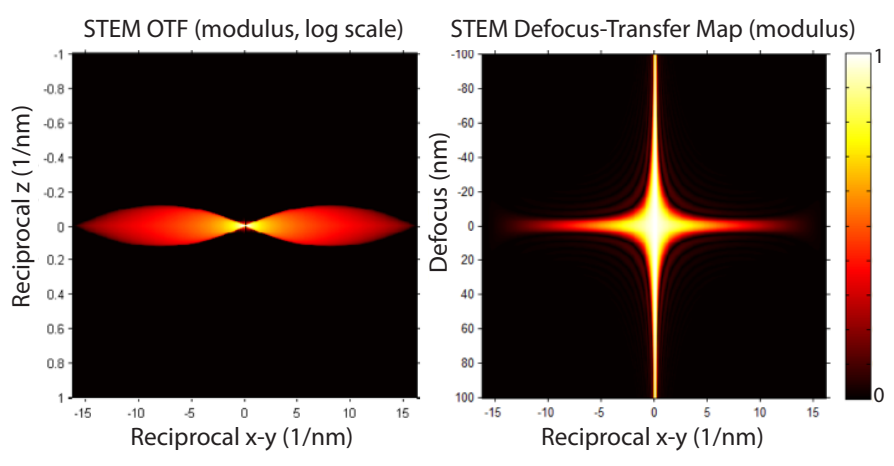

Figure 4: STEM 3D optical transfer function and defocus-transfer map calculated for the UltraSTEM 100.

with a homogenous layer of SZO, since Sr and Zr are both heavy and only differ in atomic number by two, meaning if the Zr-rich regions did contain volumes of SZO they must be buried beneath STO. Another alternative is a homogenous layer of $\mathrm{SrZr}_{x} \mathrm{Ti}_{1-x} \mathrm{O}_{3}$ with $x>0$. However, since the $\mathrm{Sr}$ concentration in STO, SZO and in fact any $\mathrm{SrZr}_{x} \mathrm{Ti}_{1-x} \mathrm{O}_{3}$ is the same, this is inconsistent with the experimental EELS observations that the Zr-rich regions are relatively poor in Sr. Furthermore, the decrease in the Sr signal is roughly the same as that of the Ti signal in these regions as one would expect for STO on top of YSZ.

These results strongly indicate that the Zr-rich regions consist of YSZ buried beneath STO, while also highlighting the desirability of performing spectroscopic optical sectioning with atomic lateral resolution. EELS can directly distinguish the different elements through the intensity of their characteristic edges, but has not previously shown the depth sensitivity required here. Because of the large missing cone region in the STEM 3D OTF, the longitudinal resolution is equal to roughly the minimum resolved characteristic transverse length scale of the object divided by the convergence angle [11. Without atomic lateral resolution the longitudinal resolution will therefore be very poor indeed as the minimum characteristic lateral length will be the width of the layer, which even for a $2 \mathrm{~nm}$ layer gives a depth resolution of only $66.7 \mathrm{~nm}$ with the $30 \mathrm{mrad}$ convergence angle used in the present work, roughly two to three times the thickness of the film in this region.

To investigate the 3D OTF in greater detail for the particular instrument used to perform the optical sectioning, STEM probe simulations were carried out for the UltraSTEM 100 operated at $100 \mathrm{kV}$ with a $30 \mathrm{mrad}$ convergence angle using an in-house code written in the MatLab programming environment 31. To generate a threedimensional optical transfer function (3D OTF) two dimensional probe simulations were first calculated across a range of defocii. These were stacked together to yield a finely sampled three-dimensional real-space probe. The principal steps in simulating the probe at each defocus were as follows: First an aberration phase surface in anglespace was defined (all aberrations other than defocus were set to zero for the purposes of this inspection). Second this 

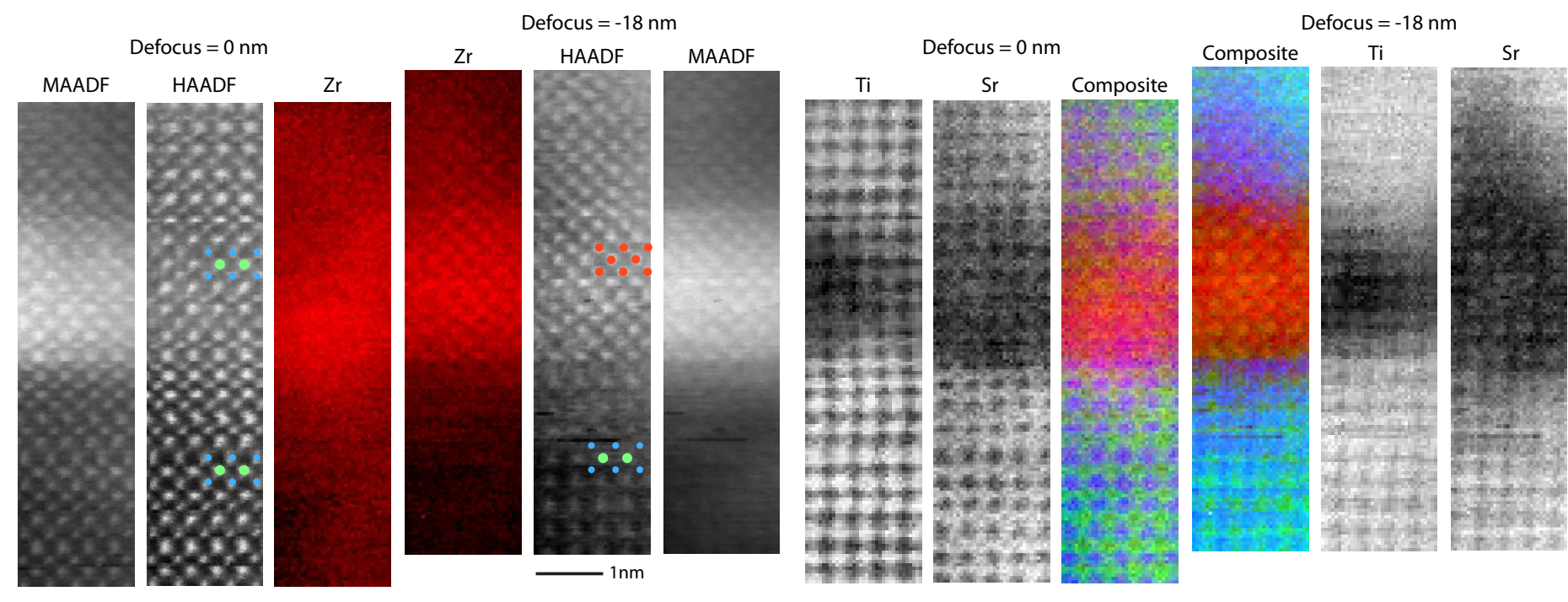

Figure 5: Simultaneous spectroscopic, HAADF and MAADF optical sectioning of a YSZ layer buried under STO in a YSZ/STO multilayer. Small overlays are again used to indicate the local composition of the heavy atoms indicated by the HAADF contrast in each slice, with $\mathrm{Sr}$ in green, $\mathrm{Ti}$ in blue and $\mathrm{Zr}$ in red. The images from the two depths are offset to best align the strain contrast seen in the MAADF.

surface was masked with a circular top-hat function to represent the objective aperture, and Fourier transformed to real-space to yield the 2D STEM probe (wholly real in this case). The 3D OTF was calculated by performing a 3D Fourier-transform on the stack of 2D STEM probes. This yields the OTF expressed in both reciprocal lateral and vertical units (perpendicular and parallel to the beam direction) as shown in Figure 4 (logarithmic colour-scale).

Alternatively, when the response of a given spatialfrequency is of interest as a function of defocus, it is convenient to express this OTF after only transforming the lateral dimension to Fourier-space. The resulting OTF has reciprocal-space lateral units (those of spatial frequency) but retains a real-space unit in the beam direction (equivalent to defocus or equally z-height). Once such a 3D OTF is calculated it can be sectioned parallel to the beam direction (vertically in Figure 4) for any given spatial frequency to yield the transfer function corresponding to sample features with the corresponding lateral spacing. We extract a depth resolution of $9 \mathrm{~nm}$ for the $0.28 \mathrm{~nm}$ minimum transverse spacing of the $\mathrm{Zr}$ lattice, only a couple of nanometers more than the $7 \mathrm{~nm}$ depth of field [10]. For the larger 0.39 $\mathrm{nm} \mathrm{Ti}-\mathrm{Ti}$ and $\mathrm{Sr}-\mathrm{Sr}$ spacings we calculate the depth resolution to be $11 \mathrm{~nm}$. Therefore EELS spectrum images acquired with sufficient transverse resolution to resolve these spacings also posses significant depth resolution.

Atomic resolution EELS spectrum images were acquired from regions appearing to contain YSZ buried beneath STO from HAADF imaging. By using the high tension fine defocus control of the UltraSTEM 100 we were able to obtain pairs of spectrum images taken in quick succes-

Figure 6: $\mathrm{Ti}$ and $\mathrm{Sr}$ and composite $(\mathrm{Ti}, \mathrm{Sr}$ and $\mathrm{Zr}$ ) chemical maps extracted from the same spectroscopic optical slices as Figure 4 of the main text. The composite maps display the $\mathrm{Sr}$ and $\mathrm{Ti}$ integrated intensities in green and blue respectively, as well as the $\mathrm{Zr}$ signal in red.

sion from the same area of the sample but with the probe focused to different depths. The Zr maps extracted from one such pair of spectrum images are displayed alongside the simultaneously acquired HAADF and MAADF images in Figure 5. The Ti and $\mathrm{Sr}$ maps from the same slices are shown in figure 6. alongside composite images of the $\mathrm{Zr}$, $\mathrm{Sr}$ and Ti signals combined together as the red, green and blue channels respectively. The optical slices were taken with the probe focused to the entrance surface and $18 \mathrm{~nm}$ into the depth of the film.

Each slice consisted of 35 by 146 EELS pixels with 0.05 second exposures. Subpixel scanning was performed in a 32 by 32 grid inside each EELS pixel to provide superior resolution in the simultaneously acquired HAADF and MAADF images. The principle component analysis method of 32 was used to eliminate noise in the atomicresolution EELS elemental maps as described in [33. For the Ti maps we again used the L-edge, however when extracting the $\mathrm{Sr}$ and $\mathrm{Zr}$ elemental maps for the optical sectioning we utilised the $\mathrm{Sr}$ and $\mathrm{Zr}$ M-edges rather than their L-edges due to the proximity of the M-edges to the Ti Ledge, which was needed to record all the edges at once on the smaller CCD of the Enfina spectrometer, and also because of their higher cross sections. The Ti L-edge fine structure was used to fine tune the calibration of the energy scale. Although the Sr and Zr M-edges are extended and overlap, they are offset in energy and have fine structure features appearing at different energies. The $\mathrm{Zr} \mathrm{M}$ edge has a pair of peaks appearing between 330 and 355 $\mathrm{eV}$ where the Sr M-edge has no fine structure and only a decaying tail, and the Sr M-edge has a pair of peaks between 270 and $286 \mathrm{eV}$ where the $\mathrm{Zr}$ edge is relatively flat. For the $\mathrm{Zr}$ edge, background subtraction fitting was performed in the flat region just after the pair of peaks at 
the Sr M-edge maximum. For the Sr edge the background fitting was performed between 218 and $240 \mathrm{eV}$, which balances the falloff of one peak in the $\mathrm{Zr}$ edge with the rise of the broad peak appearing just before the Sr integration window.

Clear differences are seen between the two spectroscopic optical slices. In the slice taken at the entrance surface of the sample (0 nm defocus), the HAADF image shows the alternating pattern of bright and dark columns associated with $\mathrm{Sr}$ and $\mathrm{Ti}$ columns in STO from top to bottom, and the Zr map reveals the presence of a Zr containing region but without any lattice contrast. However in the slice taken with the probe focused $18 \mathrm{~nm}$ further into the depth of the sample (-18 nm defocus) in the same area, a region of YSZ like contrast appears in the upper portion of the HAADF image, with all neighbouring columns having similar intensity. Here the $\mathrm{Zr}$ signal also sharpens into a lattice with precisely the periodicity expected for YSZ (compare to the overlaid Zr lattice model in Figure 5). This demonstrates spectroscopic depth sensitivity with EELS. The Zr map at zero defocus is an out of focus version of the lattice seen at $-18 \mathrm{~nm}$ defocus. We note that this lattice cannot simply be an artefact of the PCA treatment rather than depth sensitivity as if this were the case the lattice would also appear at zero defocus.

The Sr and Ti elemental maps (Figure 6) show the opposite dependence on defocus, exhibiting clearer lattice contrast at the entrance surface than at depth, again in agreement with the contrast seen in the HAADF images. Furthermore the location of the Zr lattice correlates with the appearance of high strain contrast seen in the simultaneously acquired MAADF images, which is also consistent with the presence of YSZ strained to match the STO lattice. Interestingly the strain contrast appears to be relatively independent of depth, providing a convenient reference point for drift compensation.

As seen from the data in Figure 1, the YSZ islands are not of uniform shape, and most likely not of uniform thickness based on the nonuniform intensities seen in both the $\mathrm{ADF}$ and $\mathrm{Zr}$ signals. This nonuniformity is again seen in the spectroscopic optical slices shown in Figures 5 and 6 . For instance at $-18 \mathrm{~nm}$ defocus, the $\mathrm{Zr}$ signal is strongest in the middle section of the slice, but the $\mathrm{Zr}$ lattice can be seen to continue to the top of the slice, just at a reduced intensity, consistent with the volume of YSZ being thickest in the middle of the slice and decreasing towards the top. Looking below the middle section of the slice the intensity of the Zr signal drops off much more rapidly. Although there could be a thin residual zirconia tail extending slightly further down the slice, the interface on the lower side appears to be overall relatively abrupt. This interpretation is consistent with the HAADF image from this slice, which shows STO like contrast in the bottom part of the image starting immediately below the position of the last row of strong $\mathrm{Zr}$ columns in the $\mathrm{Zr}$ map. Following the pattern of STO contrast up from the bottom part of the HAADF image, the plane below the last strong
Zr plane corresponds to a darker Ti row. The composite spectrum image also shows a row of blue Ti filled columns immediately below the last plane of strongly red $\mathrm{Zr}$ rich columns, consistent with this termination.

In the $\mathrm{Zr}$ rich regions, some contrast remains in the $\mathrm{Sr}$ and Ti maps in the optical slice taken with the probe focused to $-18 \mathrm{~nm}$. This is most likely due to both the poorer depth resolution for the larger $\mathrm{Sr}-\mathrm{Sr}$ and $\mathrm{Ti}-\mathrm{Ti}$ separations and simply that the STO occupies a greater proportion of the sample thickness than the YSZ in this region. This is supported by the Z-contrast focal series which shows clear STO like contrast over a significantly larger defocus range than clear YSZ like contrast. Hence when the probe is focused to depths at which YSZ is present some part of the STO above the YSZ remains in focus.

Ideally one would like to collect a large number of spectroscopic optical slices. With the present sample however this was not possible due to its limited dose tolerance. Even a third slice was not possible without causing damage to the sample. Nevertheless the present results are the first to show depth dependence in EELS, and two slices are already enough to reveal inhomogenaities in the atomic scale composition and structure that occur in the depth of the sample. Aside from the dose tolerance of a given sample, as is normal with EELS, the technique depends on the availability of spectral features with high enough cross sections to achieve sufficient signal to noise ratios. As the same principle should apply to energy dispersive $\mathrm{x}-$ ray spectroscopy (EDX), the additional spectroscopic features EDX is sensitive to should help expand the range of elements for which spectroscopic optical sectioning can be performed. Regardless of whether one uses EELS or EDX, the attainable depth resolution will depend on the lateral spatial frequencies present in the sample as well as the size of the convergence angle available on the microscope imaging the sample.

Lattice spacings between 1-4 $\AA$ are quite typical, and thus one can expect EELS optical sectioning to be useful for a wide range of materials. Although the depth resolution of the 3D spectrum imaging demonstrated here is not quite as high as that obtained using electron tomography [34, depth sectioning with EELS in the aberration corrected STEM provides significantly higher lateral resolution, allowing one to observe changes in atomic scale structure and composition. Furthermore, as aberration correctors become able to correct out to larger angles, the convergence angle can be further increased increasing both lateral and depth resolution. X-ray tomography has been used to perform 3D elemental mapping, but the wavelengths of x-rays along with the poor quality of x-ray lenses limit the resolution to several tens of nanometers 35]. Atom probe tomography can detect and identify the individual atoms in a 3D structure, however samples must first be formed into extremely sharp needles, the sampled volume is limited to about $10^{6} \mathrm{~nm}^{3}$ (100 nm in diameter by $100 \mathrm{~nm}$ in depth) and data collection takes hours [36, 13]. Furthermore, EELS optical sectioning offers the 
prospect of probing electronic structure in 3D by studying near edge fine structure.

\section{Conclusion}

In conclusion, we have demonstrated the ability to perform 3D elemental mapping by optical sectioning with a combination of atomic resolution EELS and Z-contrast imaging in STEM, without the need for complicated and expensive confocal configurations. The results show that what at first appear from $2 \mathrm{D}$ imaging to be regions of a $\mathrm{Zr}$ containing perovskite are in fact volumes of YSZ buried within STO, emphasising the importance of considering the possibility of $3 \mathrm{D}$ inhomogeneity. Optical sectioning with EELS significantly expands the ability of STEM to determine $3 \mathrm{D}$ changes in structure and composition, extending the advantages of 2D EELS maps into the third dimension.

\section{Acknowledgements}

This research was supported by the EPSRC through the UK National Facility for Aberration-Corrected STEM (SuperSTEM). Research at Oak Ridge National Laboratory was sponsored by the Division of Materials Sciences and Engineering of the U.S. department of Energy. Research supported by the ERC Starting Investigator Award STEMOX 239739 (MC). Work at UCM supported by MICINN through grants MAT2011-27470-C02 and Consolider Ingenio 2010 - CSD2009-00013 (Imagine).

\section{References}

[1] Y.-M. Kim, J. He, M. D. Biegalski, H. Ambaye, V. Lauter, H. M. Christen, S. T. Pantelides, S. J. Pennycook, S. V. Kalinin, A. Y. Borisevich, Probing oxygen vacancy concentration and homogeneity in solid-oxide fuel-cell cathode materials on the subunit-cell level, Nat Mater 11 (10) (2012) 888-894.

[2] S. Wang, A. Y. Borisevich, S. N. Rashkeev, M. V. Glazoff, K. Sohlberg, S. J. Pennycook, S. T. Pantelides, Dopants adsorbed as single atoms prevent degradation of catalysts, Nature Materials 3 (3) (2004) 143-146.

[3] K. van Benthem, A. R. Lupini, M. Kim, H. S. Baik, S. Doh, J.H. Lee, M. P. Oxley, S. D. Findlay, L. J. Allen, J. T. Luck, S. J. Pennycook, Three-dimensional imaging of individual hafnium atoms inside a semiconductor device, Applied Physics Letters 87 (3) (2005) 034104.

[4] A. Y. Borisevich, A. R. Lupini, S. J. Pennycook, Depth sectioning with the aberration-corrected scanning transmission electron microscope, Proceedings of the National Academy of Sciences of the United States of America 103 (9) (2006) 3044-3048.

[5] K. van Benthem, A. R. Lupini, M. P. Oxley, S. D. Findlay, L. J. Allen, S. J. Pennycook, Three-dimensional ADF imaging of individual atoms by through-focal series scanning transmission electron microscopy, Ultramicroscopy 106 (11-12) (2006) 10621068.

[6] K. Benthem, S. J. Pennycook, Imaging and spectroscopy of defects in semiconductors using aberration-corrected STEM, Applied Physics A 96 (1) (2008) 161-169.

[7] V. Ortalan, A. Uzun, B. C. Gates, N. D. Browning, Direct imaging of single metal atoms and clusters in the pores of dealuminated HY zeolite, Nature Nanotechnology 5 (7) (2010) 506-510.
[8] P. B. Hirsch, J. G. Lozano, S. Rhode, M. K. Horton, M. A. Moram, S. Zhang, M. J. Kappers, C. J. Humphreys, A. Yasuhara, E. Okunishi, P. D. Nellist, The dissociation of the [a+ c] dislocation in GaN, Philosophical Magazine 93 (2013) 39253938.

[9] J. G. Lozano, H. Yang, M. P. Guerrero-Lebrero, A. J. D'Alfonso, A. Yasuhara, E. Okunishi, S. Zhang, C. J. Humphreys, L. J. Allen, P. L. Galindo, P. B. Hirsch, P. D. Nellist, Direct Observation of Depth-Dependent Atomic Displacements Associated with Dislocations in Gallium Nitride, Phys Rev Lett 113 (13) (2014) 135503.

[10] H. Yang, J. G. Lozano, T. J. Pennycook, L. Jones, P. B. Hirsch, P. D. Nellist, Imaging screw dislocations at atomic resolution by aberration-corrected electron optical sectioning, Nat Comms 6 (2015) 7266

[11] G. Behan, E. C. Cosgriff, A. I. Kirkland, P. D. Nellist, Threedimensional imaging by optical sectioning in the aberrationcorrected scanning transmission electron microscope, Philosophical Transactions of the Royal Society a-Mathematical Physical and Engineering Sciences 367 (1903) (2009) 3825-3844.

[12] P. D. Nellist, P. Wang, Optical Sectioning and Confocal Imaging and Analysis in the Transmission Electron Microscope, Annual review of materials research 42 (1) (2012) 125-143.

[13] P. Wang, G. Behan, M. Takeguchi, A. Hashimoto, K. Mitsuishi, M. Shimojo, A. I. Kirkland, P. D. Nellist, Nanoscale Energy-Filtered Scanning Confocal Electron Microscopy Using a Double-Aberration-Corrected Transmission Electron Microscope, Physical Review Letters 104 (20) (2010) 200801.

[14] A. J. D'Alfonso, S. Findlay, M. P. Oxley, S. J. Pennycook, K. van Benthem, L. J. Allen, Depth sectioning in scanning transmission electron microscopy based on core-loss spectroscopy, Ultramicroscopy 108 (1) (2007) 17-28.

[15] L. J. Allen, S. Findlay, M. P. Oxley, M. Bosman, V. J. Keast, E. C. Cosgriff, G. Behan, P. D. Nellist, A. I. Kirkland, Theoretical interpretation of electron energyloss spectroscopic images, AIP Conf. Proc. 999 (2008) 32.

[16] A. J. D'Alfonso, E. C. Cosgriff, S. Findlay, A. I. Kirkland, P. D. Nellist, M. P. Oxley, L. J. Allen, Depth sectioning using electron energy loss spectroscopy, Journal of Physics: Conference Series 126 (2008) 012037.

[17] J. Garcia-Barriocanal, A. Rivera-Calzada, M. Varela, Z. Sefrioui, E. Iborra, C. Leon, S. J. Pennycook, J. Santamaria, Colossal Ionic Conductivity at Interfaces of Epitaxial $\mathrm{ZrO} 2: \mathrm{Y} 2 \mathrm{O} 3 / \mathrm{SrTiO} 3$ Heterostructures, Science 321 (5889) (2008) 676-680.

[18] J. Garcia-Barriocanal, A. Rivera-Calzada, M. Varela, Z. Sefrioui, E. Iborra, C. Leon, S. J. Pennycook, J. Santamaria, Response to Comment on" Colossal Ionic Conductivity at Interfaces of Epitaxial ZrO2: Y2O3/SrTiO3 Heterostructures", Science 324 (5926) (2009) 465.

[19] X. Guo, Comment on "Colossal Ionic Conductivity at Interfaces of Epitaxial $\mathrm{ZrO} 2: \mathrm{Y} 2 \mathrm{O} 3 / \mathrm{SrTiO} 3$ Heterostructures", Science 324 (5926) (2009) 465-465.

[20] A. Cavallaro, M. Burriel, J. Roqueta, A. Apostolidis, A. Bernardi, A. Tarancon, R. Srinivasan, S. N. Cook, H. L. Fraser, J. A. Kilner, D. W. McComb, J. Santiso, Electronic nature of the enhanced conductivity in YSZ-STO multilayers deposited by PLD, Solid State Ionics 181 (13-14) (2010) 592601.

[21] T. J. Pennycook, M. J. Beck, K. Varga, M. Varela, S. J. Pennycook, S. T. Pantelides, Origin of Colossal Ionic Conductivity in Oxide Multilayers: Interface Induced Sublattice Disorder, Physical Review Letters 104 (11) (2010) 115901.

[22] T. J. Pennycook, M. P. Oxley, J. Garcia-Barriocanal, F. Y. Bruno, C. Leon, J. Santamaria, S. T. Pantelides, M. Varela, S. J. Pennycook, Seeing oxygen disorder in YSZ/SrTiO3 colossal ionic conductor heterostructures using EELS, European Physical Journal-Applied Physics 54 (3) (2011) 345.

[23] A. Rivera-Calzada, M. R. Diaz-Guillen, O. J. Dura, G. SanchezSantolino, T. Pennycook, R. Schmidt, F. Y. Bruno, J. GarciaBarriocanal, Z. Sefrioui, N. M. Nemes, M. Garcia-Hernández, 
M. Varela, C. Leon, S. T. Pantelides, S. J. Pennycook, J. Santamaria, Tailoring Interface Structure in Highly Strained YSZ/STO Heterostructures, Advanced Materials 23 (44) (2011) 5268-5274.

[24] W. L. Cheah, M. W. Finnis, Structure of multilayer $\mathrm{ZrO} 2 / \mathrm{SrTiO} 3$, Journal of Materials Science 47 (4) (2011) 16311640.

[25] M. S. Dyer, G. R. Darling, J. B. Claridge, M. J. Rosseinsky, Chemical Bonding and Atomic Structure in Y2O3:ZrO2-SrTiO3 Layered Heterostructures, Angewandte Chemie International Edition 51 (14) (2012) 3418-3422.

[26] S. J. Pennycook, H. Zhou, M. F. Chisholm, A. Y. Borisevich, M. Varela, J. Gazquez, T. J. Pennycook, J. Narayan, Misfit accommodation in oxide thin film heterostructures, Acta Materialia 61 (8) (2013) 2725-2733.

[27] F. Li, R. Lu, H. Wu, E. Kan, C. Xiao, K. Deng, D. E. Ellis, The strain effect on colossal oxygen ionic conductivity in nanoscale zirconia electrolytes: a first-principles-based study, Physical Chemistry Chemical Physics 15 (8) (2013) 2692.

[28] O. L. Krivanek, G. J. Corbin, N. Dellby, B. F. Elston, R. J. Keyse, M. F. Murfitt, C. S. Own, Z. S. Szilagyi, J. W. Woodruff, An electron microscope for the aberration-corrected era, Ultramicroscopy 108 (3) (2008) 179-195.

[29] N. Dellby, N. J. Bacon, P. Hrncirik, M. F. Murfitt, G. S. Skone, Z. S. Szilagyi, O. L. Krivanek, Dedicated STEM for 200 to $40 \mathrm{keV}$ operation, European Physical Journal-Applied Physics 54 (3) (2011) 33505.

[30] C. Koch, Determination of core structure periodicity and point defect density along dislocations, ph.d. thesis (2002).

[31] L. Jones, P. D. Nellist, Three-dimensional optical transfer functions in the aberration-corrected scanning transmission electron microscope, Journal of microscopy 254 (2014) 47.

[32] M. Bosman, M. Watanabe, D. T. L. Alexander, V. J. Keast, Mapping chemical and bonding information using multivariate analysis of electron energy-loss spectrum images, Ultramicroscopy 106 (11-12) (2006) 1024-1032.

[33] M. Varela, M. P. Oxley, W. Luo, J. Tao, M. Watanabe, A. R. Lupini, S. T. Pantelides, S. J. Pennycook, Atomic-resolution imaging of oxidation states in manganites, Physical Review B 79 (8) (2009) 085117.

[34] P. A. Midgley, M. Weyland, 3D electron microscopy in the physical sciences: the development of Z-contrast and EFTEM tomography, Ultramicroscopy 96 (3-4) (2003) 413-432.

[35] B. P. Flannery, H. W. Deckman, W. G. Roberge, K. L. D'Amico, Three-Dimensional X-ray Microtomography, Science 237 (4821) (1987) 1439-1444.

[36] T. F. Kelly, M. K. Miller, Invited Review Article: Atom probe tomography, Review of Scientific Instruments 78 (3) (2007) 031101-031101-20. 